\title{
Cutibacterium (formerly Propionibacterium) acnes clavicular infection
}

\author{
Musa Zaid, Madisyn R. Chavez, Adrianna E. Carrasco, Melissa N. Zimel, Alan L. Zhang, Andrew E. \\ Horvai, Thomas M. Link, Richard J. O’Donnell ${ }^{\bowtie}$ \\ University of California San Francisco, Department of Orthopaedic Surgery \\ $\bowtie$ Corresponding author: Richard J. O'Donnell, MD. Address: 1825 Fourth Street, $4^{\text {th }}$ Floor, San Francisco, CA 94158. E-mail: Richard.O'Donnell@ucsf.edu \\ (c) Ivyspring International Publisher. This is an open access article distributed under the terms of the Creative Commons Attribution (CC BY-NC) license \\ (https://creativecommons.org/licenses/by-nc/4.0/). See http://ivyspring.com/terms for full terms and conditions.
}

Received: 2018.08.10; Accepted: 2018.10.03; Published: 2019.01.29

\begin{abstract}
Cutibacterium (formerly Propionibacterium) $a_{c n e s^{13}}{ }^{16}$ is a slow growing, gram-positive bacteria that is naturally found in higher concentrations as skin flora on the chest and back, as well as in other areas with greater numbers of hair follicles. ${ }^{25,} 37$ Most of the reported cases of $C$. acnes shoulder girdle infection follow arthroplasty surgery, ${ }^{18,20,26,27,32,35}$ which then often requires debridement, administration of intravenous antibiotics, and surgical revision of the implanted device. ${ }^{12,15,21,28-30}$ In a recent study, $56 \%$ of 193 shoulder revisions had a positive culture, $70 \%$ of which grew C. acnes. ${ }^{30}$ Despite the relatively common presumed association of $C$. acnes humeral osteomyelitis with prosthetic infection, infection of the scapula or clavicle secondary to $C$. acnes is rare. $4,23,36$ Osteomyelitis of the clavicle involving any organism is also an uncommon event that can arise spontaneously via presumed hematogenous spread, or secondary to open fractures or internal fixation. 6, $33^{3}$ The most commonly found organism in clavicular osteomyelitis is Staphylococcus aureus. ${ }^{9}$ We here report two cases of clavicular infection secondary to $C$. acnes that were not associated with implants.
\end{abstract}

Key words: Cutibacterium acnes; Propionibacterium acnes; osteomyelitis; clavicle; debridement; infection; shoulder

\section{Case Series}

Case 1. A 41 year-old right hand dominant woman presented with right medial clavicular pain of 7 months' duration. Other than a prior history of intravenous vitamin $C$ injections at a health clinic and a remote history of an entomoeba histolytica infection, she had no significant risk factors for infection nor was she immunocompromised. She recalled no specific trauma, and she had no definite predisposing conditions for the development of osteomyelitis. She had no previous history of documented shoulder girdle surgery, instrumentation, or injections. Plain radiographs of the clavicle initially demonstrated no definite abnormality (Fig. 1), and the patient was treated conservatively by her local physicians. Her erythrocyte sedimentation rate (ESR) was $49 \mathrm{~mm} / \mathrm{hr}$ and her C-reactive protein (CRP) level was 36.7 $\mathrm{mg} / \mathrm{dL}$. Repeat radiographs 4 months later showed marked progression of an expansile lytic process with cortical erosion (Fig. 2). A clavicular magnetic resonance imaging (MR) scan showed a $4 \mathrm{~cm}$ bone lesion with substantial edema of the surrounding soft tissues (Fig. 3). Her fluorine-18 fluorodeoxyglucose positron emission tomography-computed tomography (PET-CT) scan showed hypermetabolism with a maximum standardized uptake value of 12.8. A fine needle aspirate biopsy suggested the diagnosis of acute osteomyelitis. With preoperative intravenous antibiotics being held, the patient underwent clavicular biopsy, curettage, burring, and debridement. Four of 5 intraoperative cultures grew C. acnes. Histologic results confirmed the presence of fibrous tissue with woven bone formation and acute 
and chronic inflammation. The patient was treated with six weeks of intravenous rocephin, followed by maintenance with oral amoxicillin. Ten weeks later, the patient developed increased clavicular pain, swelling, erythema, warmth, and tenderness. Her ESR was $42 \mathrm{~mm} / \mathrm{hr}$ and her CRP was $65.9 \mathrm{mg} / \mathrm{dL}$. Plain radiographs showed smoothly-contoured periosteal reaction over the expanded medial to mid-clavicular osteomyelitis site (Fig. 4). A repeat MR scan revealed a phlegmon within the medullary cavity of the expanded medial clavicle. Her chest CT scan demonstrated a destructive lytic lesion of the medial clavicular region with surrounding edema, consistent with osteomyelitis (Fig. 5). A CT-guided bone biopsy produced fibroconnective tissue with chronic inflammation. After withholding preoperative antibiotics, the patient underwent repeat debridement. One of two intraoperative cultures once again grew $C$. acnes. Histologic findings included acute and chronic osteomyelitis with associated fibrosis and reactive woven bone formation (Fig. 6). The patient was treated with intravenous vancomycin for 6 weeks, followed by oral amoxicillin. The patient stopped taking this medication after 2.5 months. At the time of her last follow-up visit 2-years and 3-months after her second debridement, she remained largely symptom-free, with radiographs showing healing of the smoothly-contoured expansile clavicular lesion (Fig. 7).

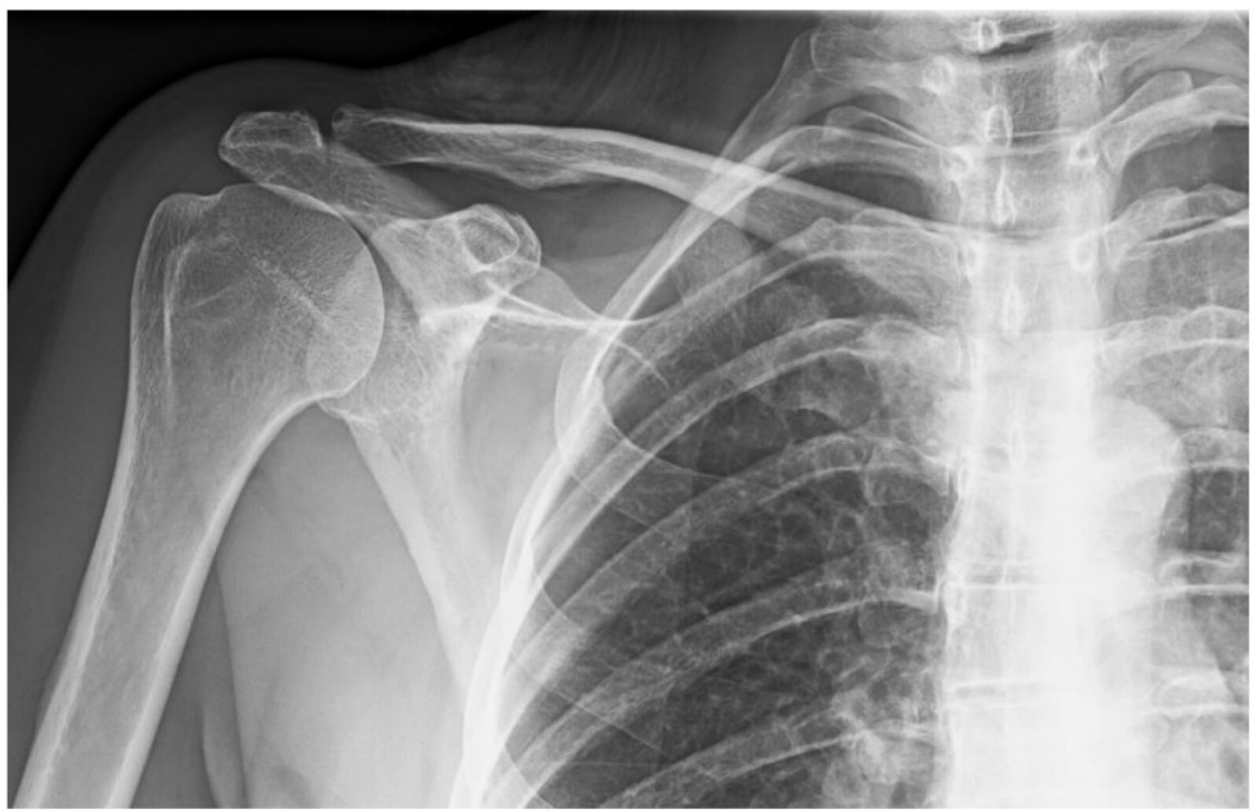

Figure 1. Anteroposterior (AP) radiograph shows an essentially normal appearance of the right clavicle.

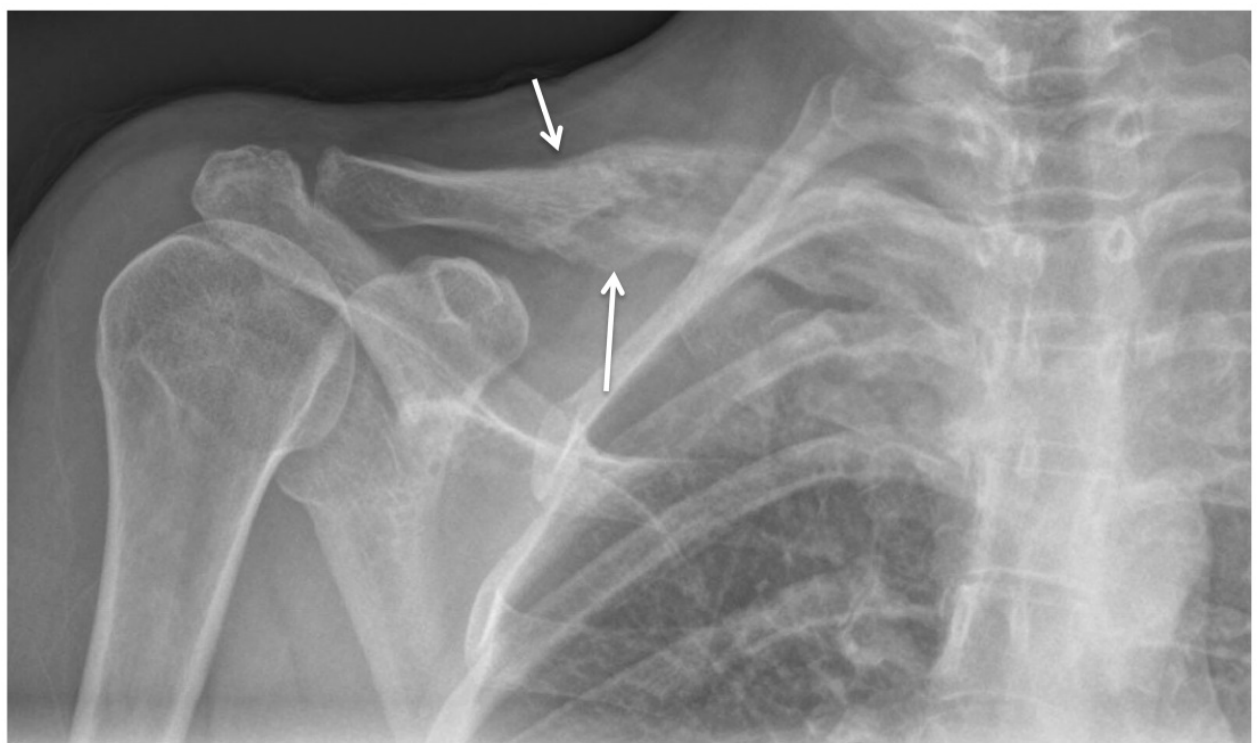

Figure 2. AP plain film demonstrates interval development of mixed lucent (long arrow) and sclerotic (short arrow) changes of the mid clavicle, with periosteal reaction that is fairly smoothly contoured. 


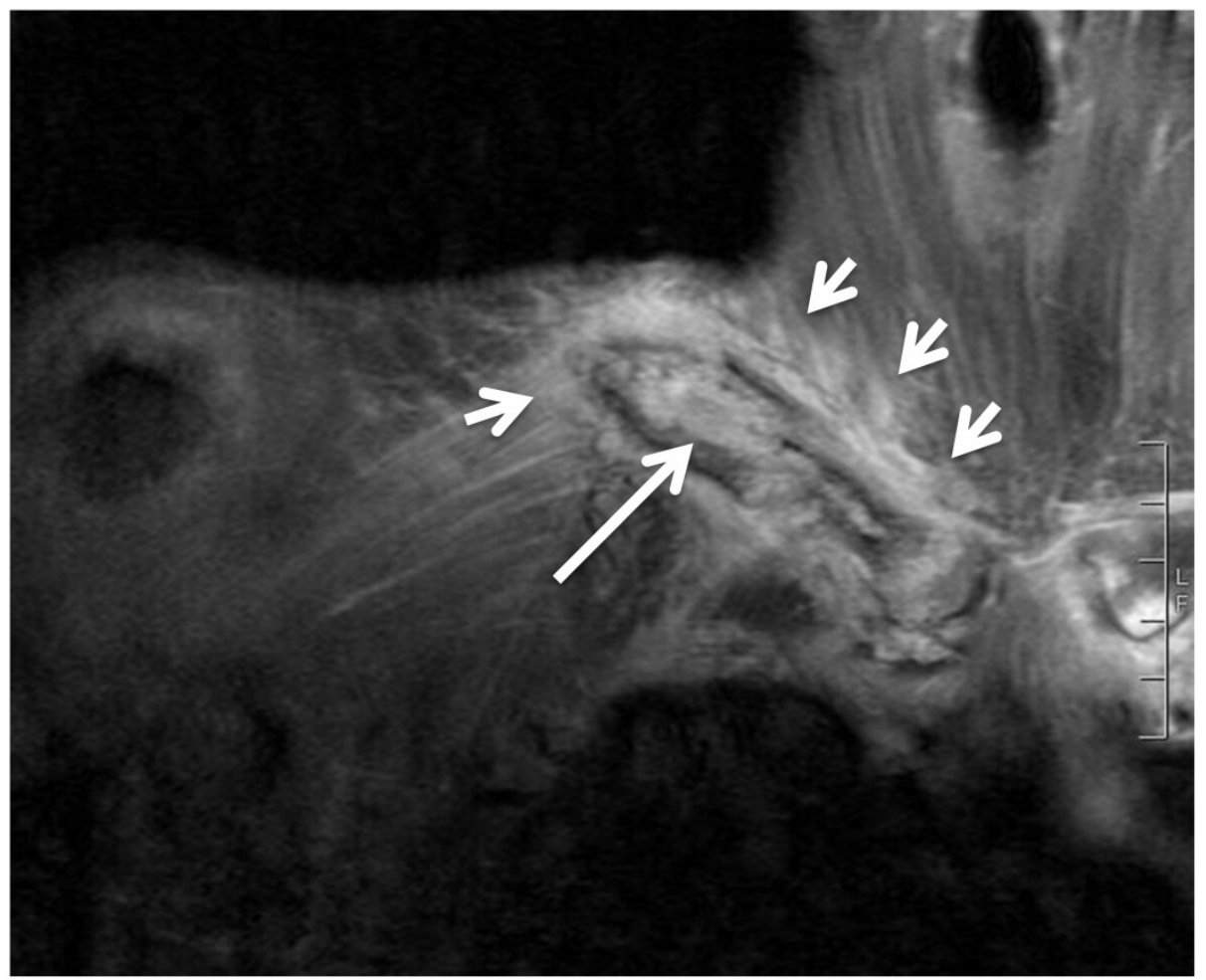

Figure 3. Coronal T2-weighted MR scan image reveals aggressive mid-clavicular lesion with hyperintense signal within the medullary cavity (long arrow) and surrounding inflammation in the soft tissues (short arrows).

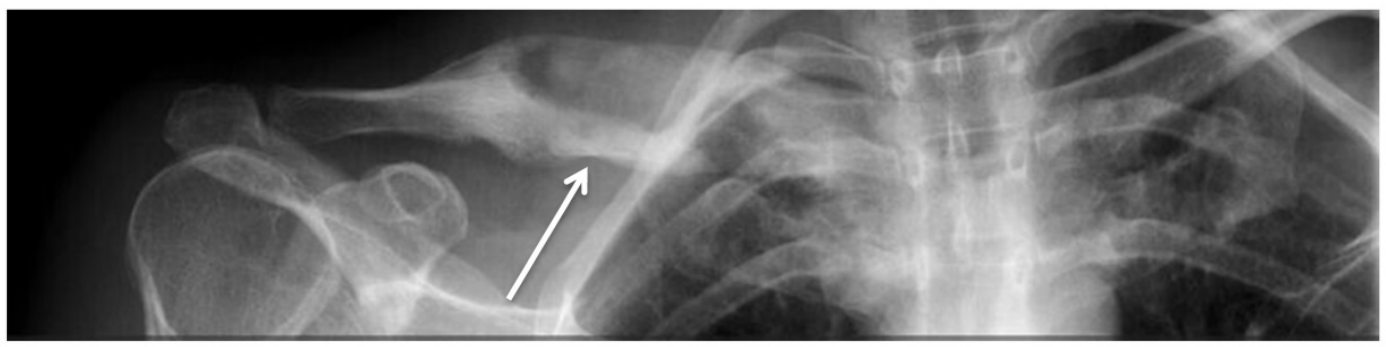

Figure 4. AP plain radiograph shows diaphyseal expansion and partial healing (arrow) in response to debridement and antibiotic treatment.

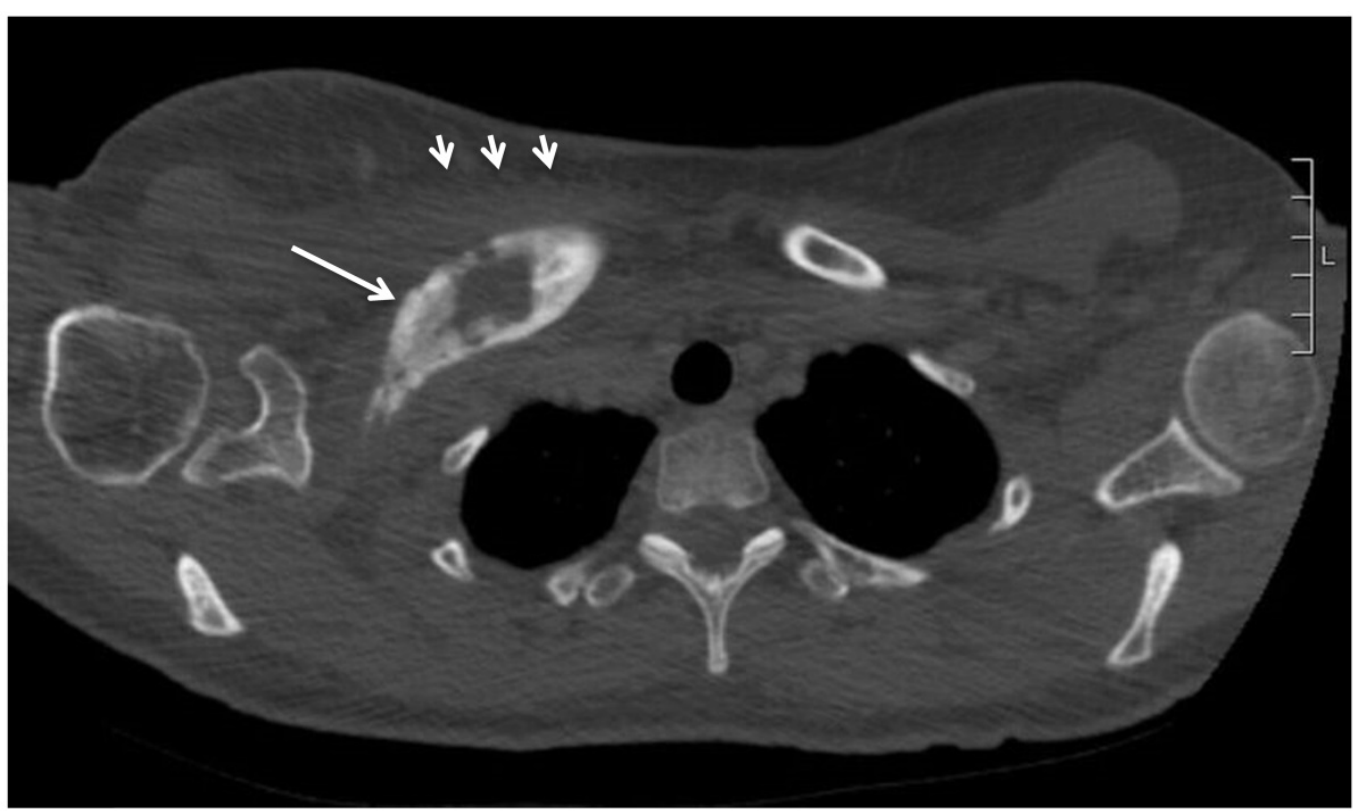

Figure 5. Axial CT image confirms clavicular expansion and reactive bone (long arrow) with surrounding soft tissue infiltration (short arrows), consistent with persistent osteomyelitis. 
a.

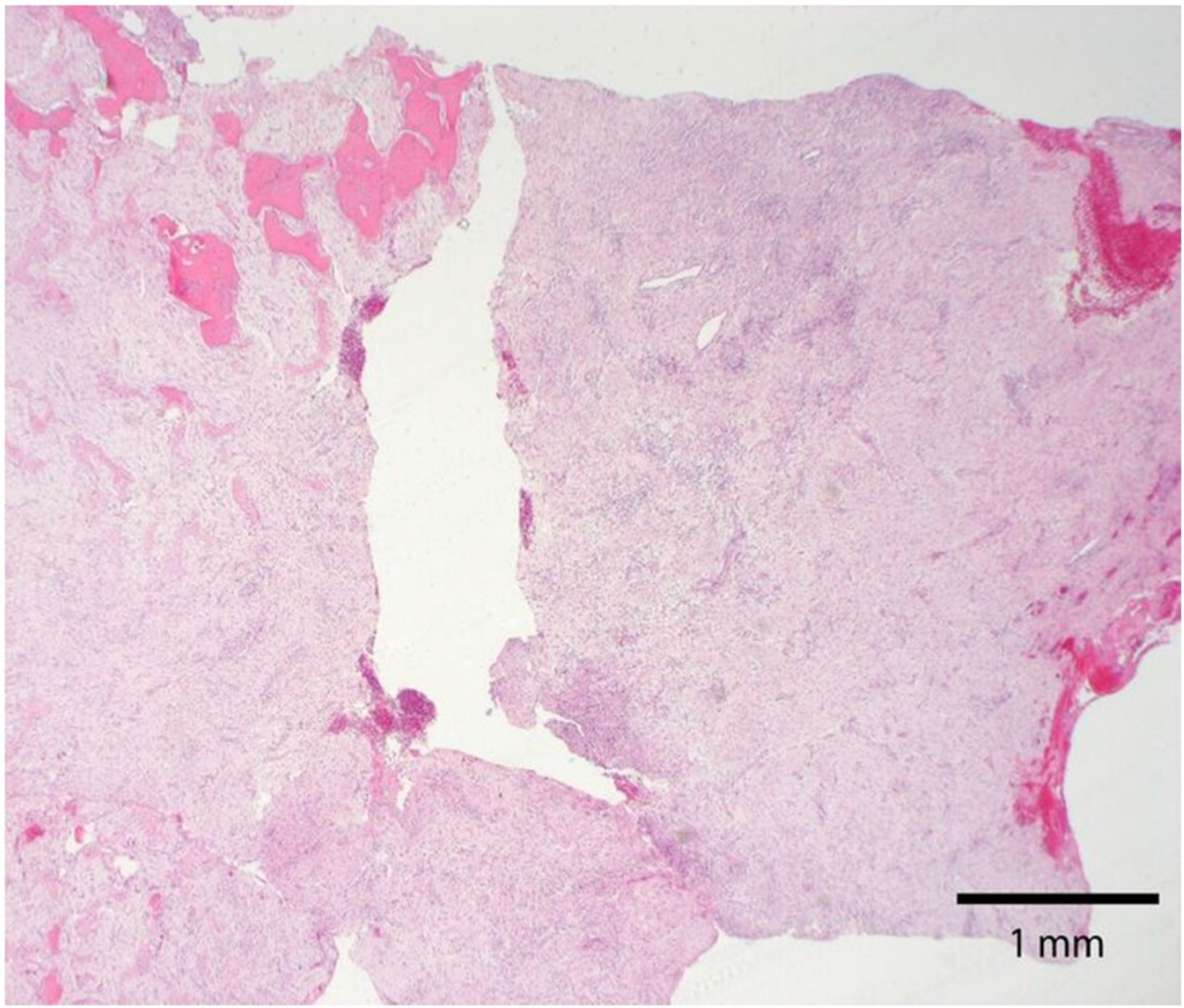

b.

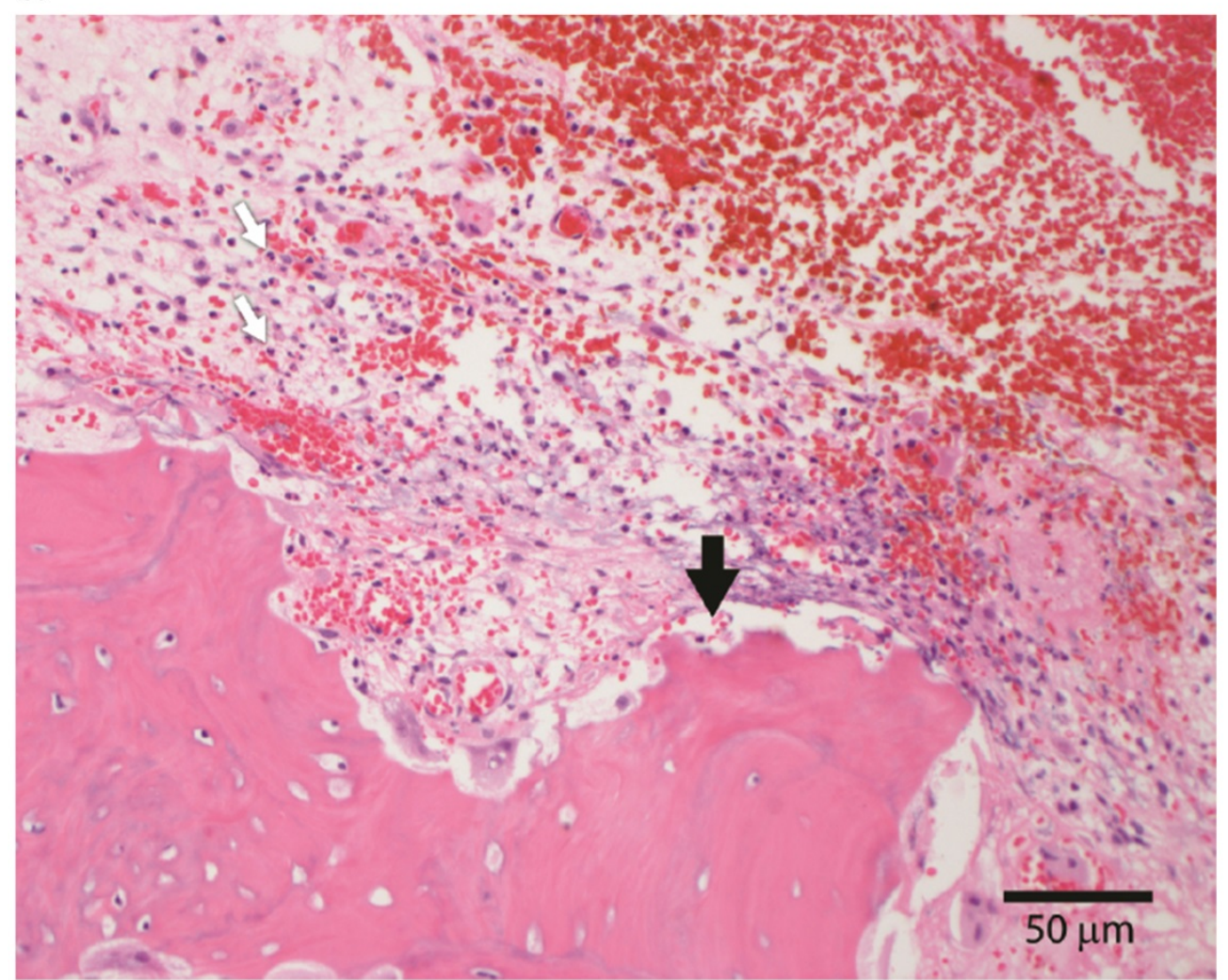

Figure 6. a. The excision specimen consisted of viable lamellar bone (top left) and new woven bone (middle left), entrapped in a fibrous matrix with vascular proliferation and extensive acute and chronic inflammation (20X original magnification). b. At higher magnification, the native lamellar bone shows extensive scalloping (black arrow) associated with a neutrophilic infiltrate (white arrows). The findings support acute osteomyelitis (200X original magnification). 


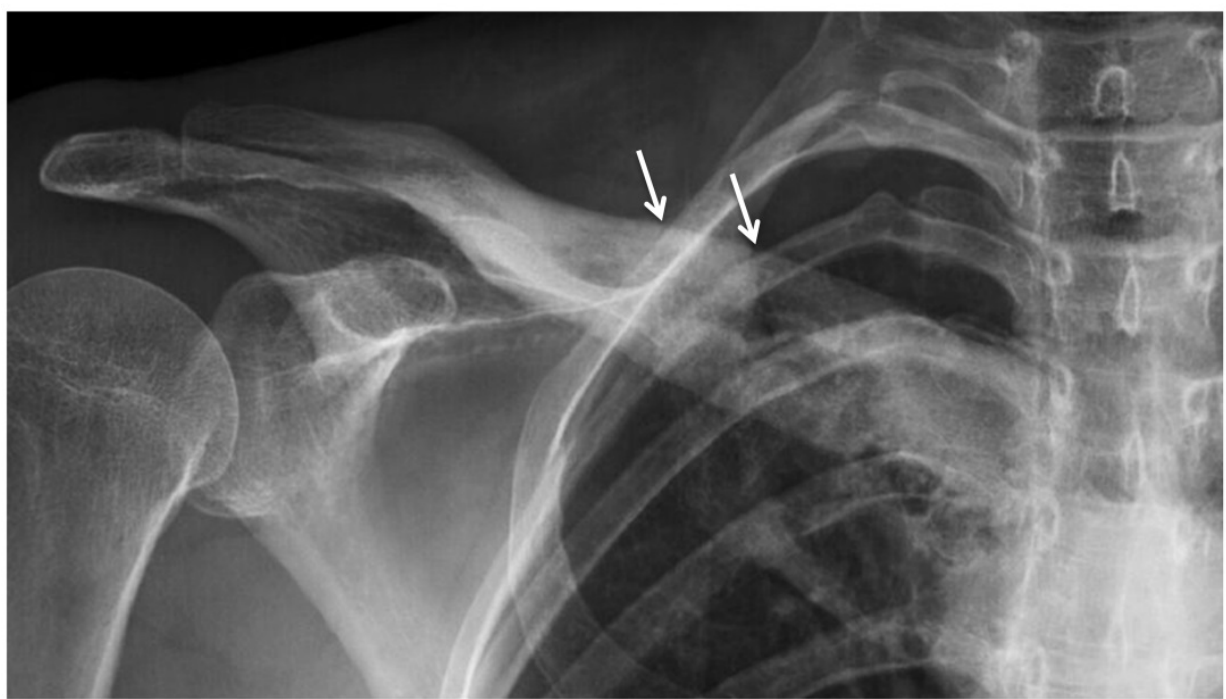

Figure 7. AP plain film more than two years after completion of treatment demonstrates improvement of appearance of the mid clavicular osteomyelitic lesion, but persistent expansion with thickened cortex (arrows).

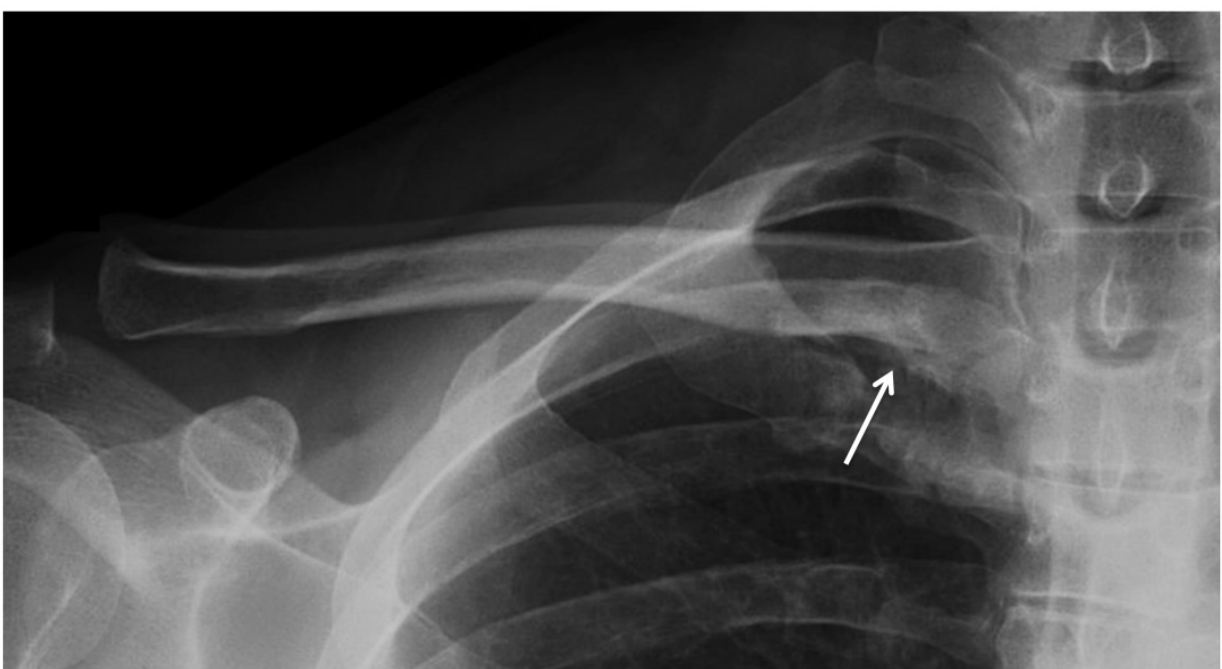

Figure 8. AP study reveals subtle medial clavicular sclerosis and inferior cortical erosion (arrow).

Case 2. A 46 year-old right hand dominant man presented with right medial clavicular pain of one year duration. His past medical history was notable for hyperthyroidism. He was not immunocompromised and he had no previous history of shoulder girdle surgery, instrumentation, or injections. He recalled no injury, and he had no definite predisposing conditions for the development of musculoskeletal infection. Laboratory studies showed an ESR of $10 \mathrm{~mm} / \mathrm{hr}$ and a CRP of $2.9 \mathrm{mg} / \mathrm{dL}$. Plain films revealed erosion and osseous remodeling of the inferior aspect of the medial clavicle (Fig. 8). A bone scan showed markedly increased uptake in the same area. A CT scan demonstrated mixed lucency and sclerosis in this region (Fig. 9). An MR scan showed medial clavicular edema extending to the sternoclavicular joint with erosive changes of the clavicle and first rib, as well as fluid collections extending anteroinferiorly from the posteromedial clavicle (Fig. 10). C. acnes grew on an ultrasound-guided soft tissue aspirate. The patient underwent incisional biopsy and debridement. Prior to obtaining surgical cultures at the time of surgical biopsy and debridement, intravenous antibiotics were held. The final pathological diagnosis was acute and chronic synovitis with fibrinopurulent exudate (Fig. 11). Three out of his four bacterial cultures grew $C$. acnes. After being maintained on intravenous ceftriaxone for six weeks, the patient presented with no further signs of infection. Follow-up at 2 years post-procedure showed that the patient was asymptomatic with normalized plain radiographs. 


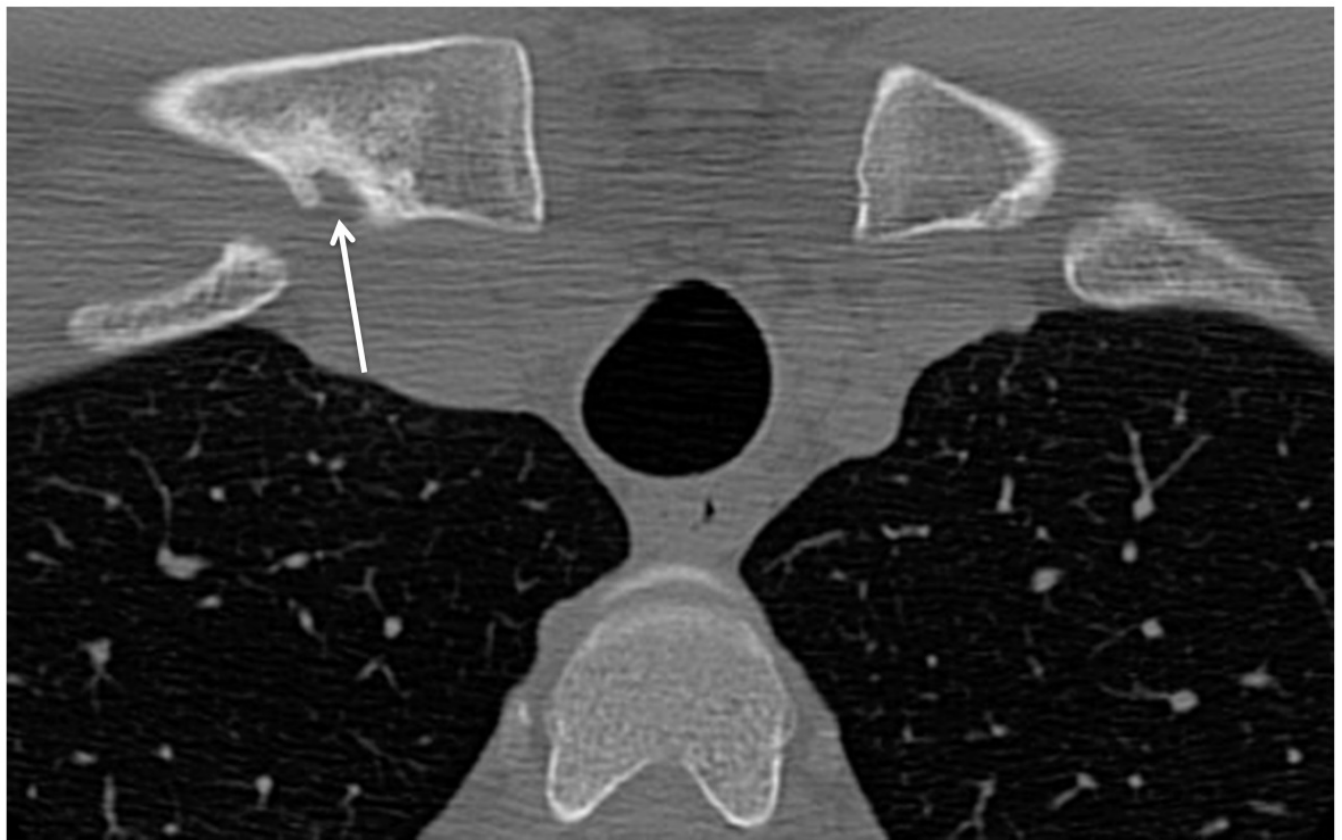

Figure 9. Axial CT image confirms mixed blastic and erosive changes along the medial clavicle (arrow)

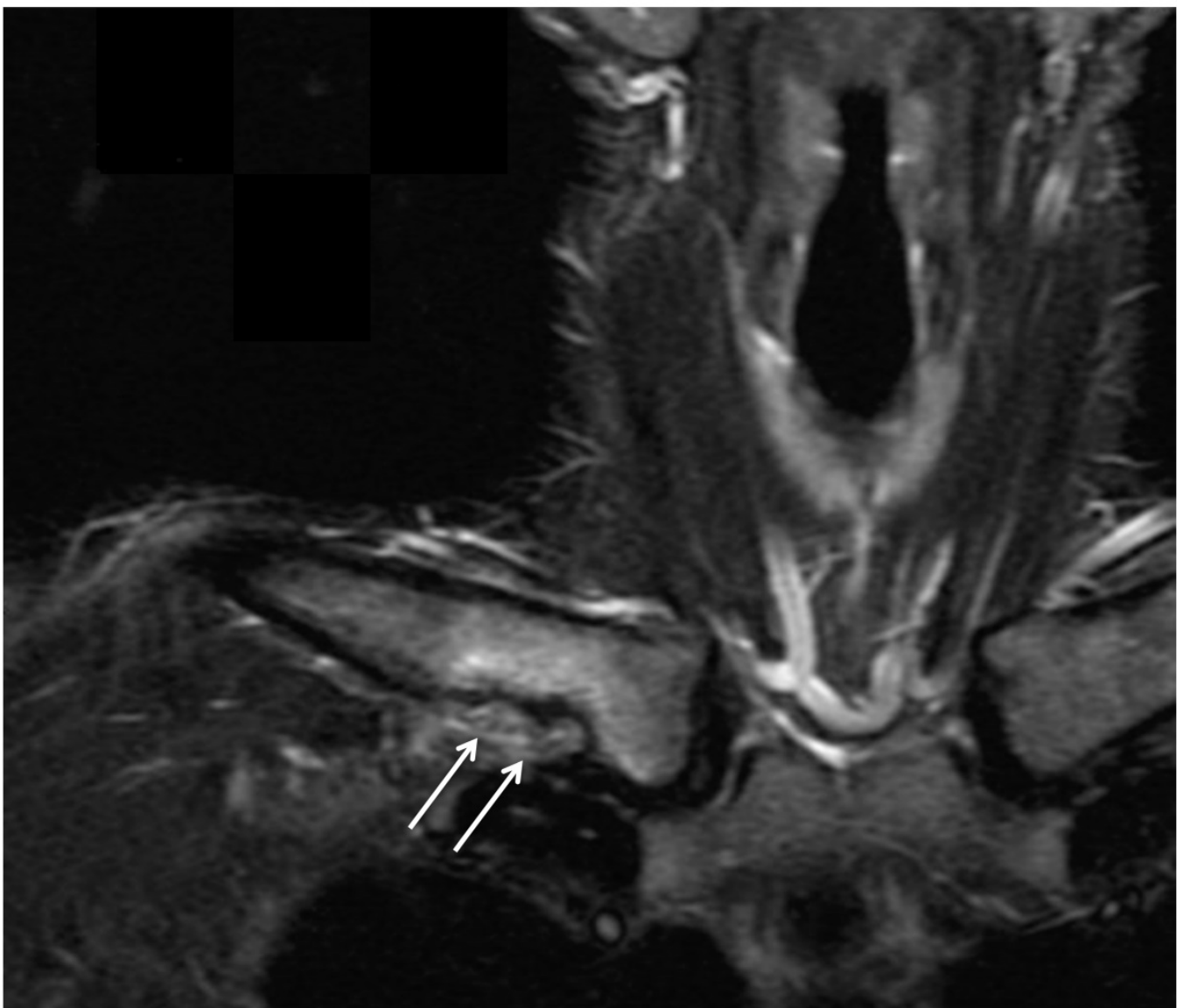

Figure 10. Coronal T2-weighted MR scan shows bone marrow edema, erosions and phlegmon inferior to medial clavicle (arrows). 
a.

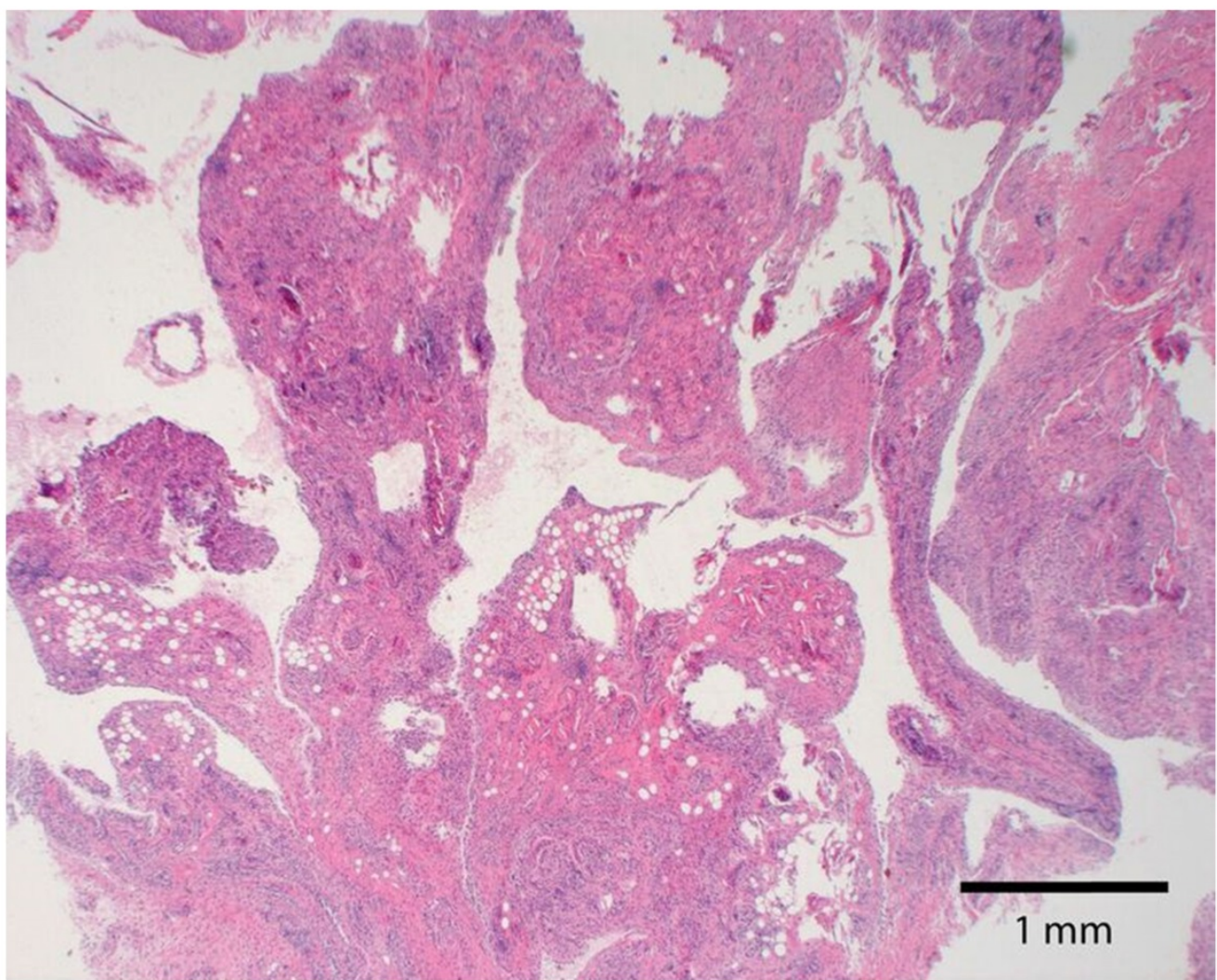

b.

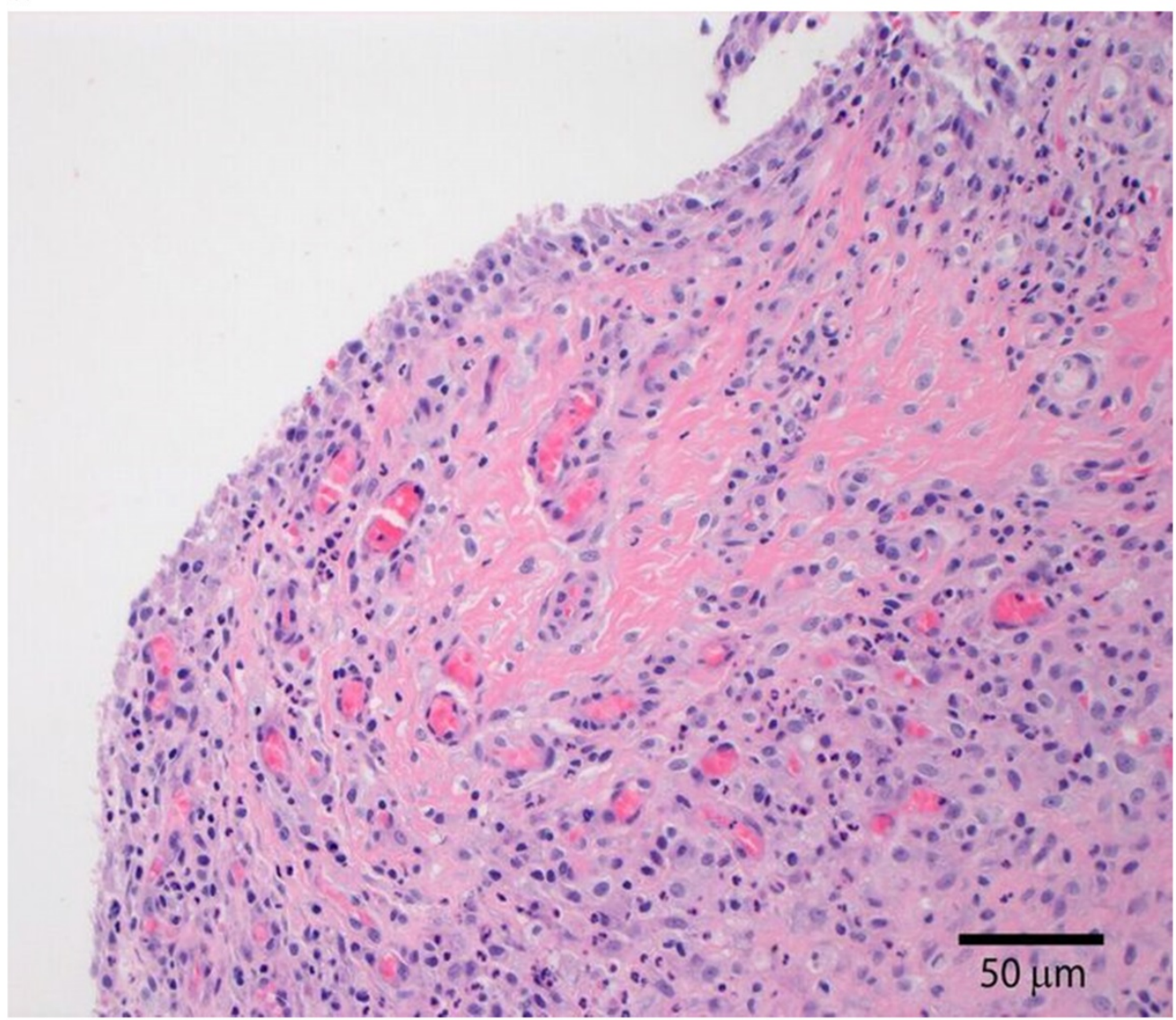


c.

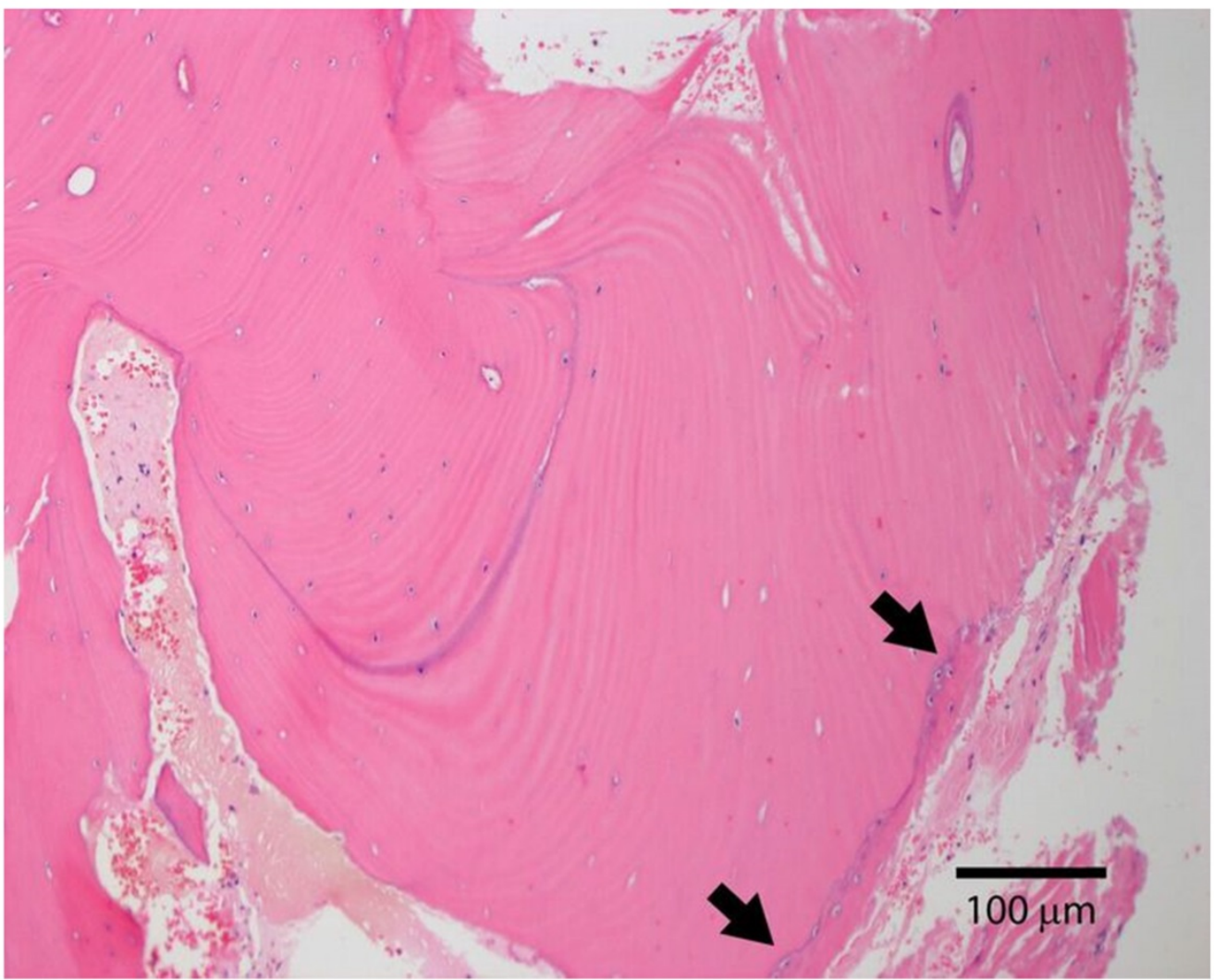

Figure 11. a. Synovium: At scanning magnification, the synovium showed slight villous hyperplasia and scant exudate (20X original magnification). b. Synovium: A moderately dense acute inflammatory infiltrate was present in the subsynovial connective tissue (200X original magnification). c. Bone: The bone was viable without significant inflammation or scalloping and thus there was no definite histologic support for acute osteomyelitis. A small focus of new, woven bone (arrows) was noted in the subperiosteal compartment, which, while nonspecific, suggested reaction to a soft tissue process (100X original magnification).

Table 1. Summary of cases at time of initial diagnosis

\begin{tabular}{|c|c|c|c|c|c|c|c|}
\hline Case & Age & Sex & Labs & Intraop Cultures & Pathology & Antibiotic Therapy & Outcome \\
\hline 1 & 41 & $\mathrm{~F}$ & $\begin{array}{l}\text { ESR: } 49 \mathrm{~mm} / \mathrm{hr} \\
\text { CRP: } 36.7 \\
\mathrm{mg} / \mathrm{dL}\end{array}$ & $4 / 5+$ C. acnes & $\begin{array}{l}\text { Fibrous tissue with woven } \\
\text { bone, acute and chronic } \\
\text { inflammation }\end{array}$ & $\begin{array}{l}\text { IV Vancomycin x } 6 \text { weeks, } \\
\text { PO Amoxicillin x } 2.5 \\
\text { months }\end{array}$ & $\begin{array}{l}\text { Asymptomatic at } 27 \text { months, } \\
\text { healing of lesion on radiographs }\end{array}$ \\
\hline 2 & 46 & M & $\begin{array}{l}\text { ESR: } 10 \mathrm{~mm} / \mathrm{hr} \\
\text { CRP: } 2.9 \\
\mathrm{mg} / \mathrm{dL}\end{array}$ & $3 / 4+$ C. Acnes & $\begin{array}{l}\text { Acute and chronic synovitis } \\
\text { with fibropurulent exudate }\end{array}$ & IV Ceftriaxone $x 6$ weeks & $\begin{array}{l}\text { Asymptomatic at } 24 \text { months, } \\
\text { normalized radiographs }\end{array}$ \\
\hline
\end{tabular}

\section{Discussion}

C. acnes is increasingly recognized as a prime causative agent of shoulder septic prosthetic arthritis and associated humeral osteomyelitis. ${ }^{21}$ 26, 29, 30, 32, 35 Multiple reports have documented the thorough and meticulous microbiologic technique needed to culture this fastidious organism when joint infection is suspected. $8,25,38$ The incidence of bone infection is thought to be much less; in fact, in an animal model, direct inoculation of $C$. acnes into the femur did not produce osteomyelitis unless an implant was present. ${ }^{34}$ Most cases of $C$. acnes osteomyelitis are understood to be associated with the spine, often in the presence of instrumentation.4,23 A case of $C$. acnes tibial osteomyelitis in association with sickle cell anemia has been reported; 5 the only previously-reported cases of C. acnes clavicular osteomyelitis were associated with internal fixation hardware. ${ }^{36}$ The two cases of clavicular infection secondary to $C$. acnes presented here demonstrate the importance of considering this organism as a causative agent in any shoulder girdle infection. Positive cultures are sometimes viewed as a "contaminant" or a "false-positive," or even reflective of "non-pathogenic" Cutibacterium "growth in deep-tissue layers," 28 but in our study, this fastidious organism grew on multiple cultures obtained on separate occasions in both patients. One patient required a second debridement procedure, which is often necessary in cases of clavicular infection. The treatment failure in this first case is likely multifactorial. C. acnes has been commonly cited an organism that is difficult to eradicate, in part because, 
the biofilm which is produced, at least in cases of implant-associated infections, ${ }^{1}$ may be protective against host immune responses and antimicrobial therapy. ${ }^{10,19}$ Additionally, bacteria in biofilm may be ultimately more difficult to effectively debride during surgery resulting in an increased residual bacterial burden. In addition to biofilm production, treatment failure in this case may be attributed to cefazolin resistance which has been previously documented in C. acnes isolates. This patient ultimately responded well to vancomycin therapy. ${ }^{31}$ It should be noted that histologic findings may not necessarily show "classic" features of acute osteomyelitis (e.g. osteonecrosis, suppurative inflammation), possibly due to sampling error. In addition to debridement, the standard of care remains treatment with intravenous antibiotics, generally for six weeks' duration, though institutional practices may differ. Further suppression with oral antibiotic management for a variable period of time might be helpful in recalcitrant cases.

The index of suspicion must remain high when considering infection as a cause for medial clavicle pain as the clinician must consider several mimicking conditions. Entities that need to be considered in the differential diagnosis in a patient with medial clavicular pain and changes seen on radiographs include condensing osteitis, Freidrich disease, sternoclavicular osteoarthritis, and chronic recurrent multifocal osteomyelitis (CRMO). Condensing osteitis of the clavicle is a rare self-limited condition characterized by sclerosis of the medial clavicular head that has been hypothesized to be traumatic or degenerative in nature. $7,11,14$ Condensing osteitis has characteristic imaging findings on radiographs including homogenous dense sclerosis most often at the inferomedial clavicle without periosteal reaction or bony destruction. Unlike c. acnes osteomyelitis, condensing osteitis is self-limited and should spontaneously resolve in months. Freidrich disease, or osteonecrosis of the medial aspect of the clavicle, is also a rare condition that may present with localized pain. Radiographically, Freidrich disease is unique as it involves the entire medial head of the clavicle, compared to just the inferior aspect as seen in condensing osteitis. ${ }^{24}$ Pathology will demonstrate necrotic bone with empty lacunae. Degenerative sternoclavicular osteoarthritis can be seen on radiographs as narrowing if the sternoclavicular joint space, with osteophytes and subchondral cysts which are often absent in condensing osteitis. ${ }^{17} \mathrm{CRMO}$ which usually affects children and adolescents, presents with pain in the effect bone, with the clavicle being a common site.22 Radiographically, CRMO presents with lytic lesions without a periosteal reaction on plain films and edematous bone marrow changes and medullary destruction on MR imaging. ${ }^{22}$ Biopsy in CRMO may demonstrate inflammatory changes, and cultures are, by definition, negative. ${ }^{22}$

\section{Conclusion}

In addition to conditions traditionally associated with sclerosis of the medial clavicle, such as condensing osteitis, osteonecrosis, sternoclavicular osteoarthritis, and (CRMO), chronic osteomyelitis must be considered. With recent advances in diagnostic testing, C. acnes infection is a growing concern for involvement in osteomyelitis. ${ }^{4,} 23$ Most cases of $C$. acnes infection arise after placement of an orthopaedic implant; however, the absence of prior surgery should not rule out $c$. acnes shoulder girdle infection. $4,5,23$ Our two cases illustrate the possibility of clavicular osteomyelitis being caused by C. acnes, without the presence of a device. Therefore, cultures of potential shoulder girdle infections that grow $C$. acnes should be taken seriously and not dismissed as contaminants; in cases of suspected prosthetic joint infection, bone specimens should be sent for tissue culture, in addition to cultures of the soft tissue and fluid. Incubation for a prolonged period (up to 14 days) is currently regarded as the best diagnostic method for identifying $C$. acnes; subculture in thioglycolate broth is believed to improve the yield of culture. Utilizing a combination of clinical and laboratory criteria $^{3,4}$ and emerging microbiologic tests $^{2}$ may help to improve the predictive value of $C$. acnes diagnostic testing in the future. The standard of care for $C$. acnes musculoskeletal infections remains adequate debridement and intravenous antibiotic administration for up to 6 weeks. High rates of susceptibility to narrow spectrum beta-lactams make this class a good initial intravenous option. Use of oral agents such as rifampin remains controversial. Treatment failures may be attributed to biofilm production which has been demonstrated to be protective against host immune response and antimicrobial therapy as well as surgical debridement or increasing rates of antimicrobial resistance. Prospective clinical studies in the area of periprosthetic joint infection will be needed to determine the optimal antimicrobial therapy for $C$. acnes.

\section{Competing Interests}

The authors have declared that no competing interest exists.

\section{References}

1. Achermann Y, Goldstein EJC, Coenye T, Shirtliff ME. Propionibacterium acnes: From commensal to opportunistic biofilm-associated implant pathogen. Clinical Microbiology Reviews. 2014;27:419-439. 
2. Achermann $Y$, Tran B, Kang M, Harro JM, Shirtliff ME. Immunoproteomic identification of In vivo-produced Propionibacterium acnes proteins in a rabbit biofilm infection model. Clinical and Vaccine Immunology. 2015;22:467-476.

3. Ahsan ZS, Somerson JS, Matsen FA III. Characterizing the Propionibacterium load in revision shoulder arthroplasty: A study of 137 culture-positive cases. Journal of Bone and Joint Surgery. 2017;99:150-154.

4. Asseray N, Papin C, Touchais S, Bemer P, Lambert C, Boutoille D et al. Improving diagnsotic criteria for Propionibacterium acnes osteomyelitis: A retrospective analysis. Scandinavian Journal of Infectious Disease. 2010;42:421-425.

5. Bachmeyer C, Blanchard M, Zeller V, Graff W, Desplaces N, Stankovic K et al. Tibial chronic osteomyelitis due to Propionibacterium acnes in a patient with sickle cell anemia. International Journal of Infectious Diseases. 2010;14S:e344.

6. Balakrishnan C, Vashi C, Jackson O, Hess J. Post-traumatic osteomyelitis of the clavicle: A case report and review of literature. Canadian Journal of Plastic Surgery. 2008;16:89-91.

7. Brower AC, Sweet DE, Keats TE. Condensing osteitis of the clavicle: A new entity. American Journal of Roentgenology, Radium Therapy, and Nuclear Medicine. 1974;121:17-21.

8. Butler-Wu SM, Burns EM, Pottinger PS, Magaret AS, Rakeman JL, Matsen FA III et al. Optimization of periprosthetic culture for diagnosis of Propionibacterium acnes prosthetic joint infection. Journal of Clinical Microbiology. 2011;49:2490-2495.

9. Calhoun JH, Manring MM, Shitliff M. Osteomyelitis of the long bones. Seminars in Plastic Surgery. 2009;23:59-72.

10. Coenye T, Peeters E, Nelis HJ. Biofilm formation by Propionibacterium acnes is associated with increased resistance to antimicrobial agents and increased production of putative virulence factors. Research in Microbiology. 2007;158:386-392.

11. Cone RO, Resnick D, Goergen TG, Robinson C, Vint V, Haghighi P. Condensing osteitis of the clavicle. AJR 1983;141:387-388.

12. Dodson CC, Craig EV, Cordasco FA, Dines DM, Dines JS, DiCarlo E et al. Propionibacterium acnes infection after shoulder arthroplasty: A diagnostic challenge. Journal of Shoulder and Elbow Surgery. 2010;19:303-307.

13. Dréno B, Pécastaings S, Corvec S, Veraldi S, Khammari A, Roques C. Cutibacterium acnes (Propionibacterium acnes) and acne vulgaris: A brief look at the latest updates. Journal of the European Academy of Dermatology and Venerology. 2018;32:5-14

14. Duró JC, Estrada P, Ribas D, Bartrons S, Rotés-Querol J. Condensing osteitis of the clavicle. Arthritis and Rheumatism. 1981;24:1454-1455.

15. Frangiamore SJ, Saleh A, Grosso MJ, Alolabi B, Bauer TW, Ianotti JP et al. Early versus late culture growth of Propionibacterium acnes in revision shoulder arthroplasty. Journal of Bone and Joint Surgery. 2015;97:1149-1158.

16. Gharamti AA, Kanafani ZA. Cutibacterium (formerly Propionibacterium) acnes infections associated with implantable devices. Expert Review of Anti-infective Therapy. 2017;15:1083-1094.

17. Greenspan A, Gerscovish E, Szabo RM, Matthews JG II. Condensing osteitis of the clavicle: A rare but frequently misdiagnosed condition. AJR. 1991 156:1011-1015

18. Hasan SS, Ricchetti ET. When is a positive culture in shoulder surgery not an infection? Commentary on an article by William R. Mook, MD, et al.: "The Incidence of Propionibacterium acnes in Open ShoulderSurgery. A Controlled Diagnostic Study". Journal of Bone and Joint Surgery. 2015;97:e51(51-53).

19. Holmberg A, Lood R, Mörgelin M, Söderquist B, Holst E, Collin M et al. Biofilm formation by Propionibacterium acnes is a characteristic of invasive isolates. Clinical Microbiology and Infection. 2009;15:787-795.

20. Hsu JE, Bumgarner RE, Matsen FA III. Propionibacterium in shoulder arthroplasty: What we think we know today. Journal of Bone and Joint Surgery. 2016;98:597-606.

21. Hsu JE, Gorbaty JD, Whitney IJ, Matsen FA III. Single-stage revision is effective for failed shoulder arthroplasty with positive cultures for Propionibacterium. Journal of Bone and Joint Surgery. 2016;98:2047-2051.

22. Iyer RS, Thapa MM, Chew FS. Chronic recurrent multifocal osteomyelitis: Review. AJR. 2011;196:S87-S91.

23. Kowalski TJ, Berbari EF, Huddleston PM, Steckelberg JM, Osmon DR. Propionobacterium acnes vertebral osteomyelitis: Seek and ye shall find? Clinical Orthopaedics and Related Research. 2007;461:25-30.

24. Levy M, Goldberg I, Fischel RE, Maor P. Friedrich's disease: Aseptic necrosis of the sternal end of the clavicle. Journal of Bone and Joint Surgery. 1981;63:539-541.

25. Matsen FA III, Butler-Wu S, Carofino BC, Jette JL, Bertelsen A, Bumgarner R. Origin of propionibacterium in surgical wounds and evidence-based approach for culturing propionibacterium from surgical sites. Journal of Bone and Joint Surgery. 2013;95:e181(181-187)

26. McGoldrick E, McElvany MD, Butler-Wu S, Pottinger PS, Matsen FA III. Substantial cultures of Propionibacterium can be found in apparently aseptic shoulders revised three years or more after the index arthroplasty. Journal of Shoulder and Elbow Surgery. 2015;24:31-35.

27. Mook WR, Klement MR, Green CL, Hazen KC, Garrigues GE. The incidence of Propionibacterium acnes in open shoulder surgery: A controlled diagnsotic study. Journal of Bone and Joint Surgery. 2015;97:957-963.

28. Moroder P, Trampuz A, Scheibel M. Propionibacterium: We found it, now we have to deal with it. Journal of Bone and Joint Surgery. 2016;98:e112(111-112).
29. Nelson GN, Davis DE, Namdari S. Outcomes in the treatment of periprosthetic joint infection after shoulder arthroplasty: A systematic review. Journal of Shoulder and Elbow Surgery. 2016;25:1337-1345.

30. Pottinger P, Butler-Wu S, Neradilek B, Merritt A, Bertelsen A, Jette JL et al. Prognostic factors for bacterial cultures positive for Propionibacterium acnes and other organisms in a large series of revision shoulder arthroplasties performed for stiffness, pain, or loosening. Journal of Bone and Joint Surgery. 2012;94:2075-2083.

31. Ramage G, Tunney MM, Patrick S, Gorman SP, Nixon J. Formation of Propionibacterium acnes biofilms on orthopaedic biomaterials and their susceptibility to antimicrobials. Biomaterials. 2003;24:3221-3227.

32. Richards J, Inacio MCS, Beckett M, Navarro RA, Singh A, Dillon MT et al. Pateint and procedure-specific risk factors for deep infection after primary shoulder arthroplasty. Clinical Orthopaedics and Related Research. 2014;472:2809-2815.

33. Saglam F, Saglam S, Gulabi D, Eceviz E, Elmail N, Yilmaz M. Bilateral clavicle osteomyelitis: A case report. International Journal of Surgery Case Reports. 2014:5:932-935.

34. Shiono Y, Ishii K, Nagai S, Kakinuma H, Sasaki A, Funao H et al. Delayed Propionibacterium acnes surgical site infections occur only in the presence of an implant. Scientific Reports. 2016;6:32758.

35. Singh JA, Sperling JW, Schleck C, Harmsen WS, Cofield RH. Periprosthetic infections after total shoulder arthroplasty: A 33-year perspective. Journal of Shoulder and Elbow Surgery. 2012;21:1534-1541.

36. von Keudell AG, Nelson SB, Jupiter JB. Propionibacterium acnes infection complicating the operative treatment of clavicle fractures. The Orthopaedic Journal at Harvard Medical School. 2015;16:66-74.

37. Wilson M. Microbial inhabitants of humans: Their ecology and role in health and disease. Cambridge University Press; 2005

38. Zhang AL, Feeley BT, Schwartz BT, Chung TT, Ma CB. Management of deep postoperative shoulder infections: Is there a role for open biopsy during surgical treatment? Journal of Shoulder and Elbow Surgery. 2015;24:e15-e20. 